\title{
Post - radiotherapy side effects for head and neck cancer - the patient's perspective
}

\author{
O. Brady, M. Donnelly, A. Horgan and M. Maher \\ Mater Private Hospital, Eccles Street, Dublin 7, Ireland
}

Malnutrition is a common complication of head and neck cancer. The nutritional support of this patient group needs to be considered for at least 6 months post treatment (Johnston et al. 1981). It is estimated that $10 \%$ of long-term head and neck cancer survivors require permanent enteral nutrition (Machtay et al. 2002). Significant functional impairment after chemoradiation has also been documented (Shilley et al. 2006). However, there is limited information in the literature regarding the long term side effects of radiation therapy to the head and neck area. The goal of this patient questionnaire was to investigate the nutritional outcomes of patients who attended for radiation treatment for head and neck cancer at the Mater Private Hospital, Dublin between 2002 and 2009.

A qualitative questionnaire was posted to 97 patients who underwent radiotherapy for head and neck cancer and who were seen by the dietitian at the Mater Private Hospital between 2002 and 2009. Of the 162 patients who were considered for the questionnaire, 51 patients had died and 14 were lost to follow-up. 54 patients returned the questionnaire and consent forms, achieving a response rate of $56 \%$. Ethics approval was granted for this study and the questionnaire was piloted on a cohort of 10 patients.

Of the 54 patients who completed the questionnaire $48 \%$ had not returned to their normal pre-radiotherapy weight at the time of questionnaire. 17 patients in this study had chemoradiation, with $53 \%$ of these patients not returning to their normal pre-radiotherapy weight. Over $82 \%$ of all patients surveyed had returned to normal diet but over a third of the patients who underwent chemoradiation had not returned to normal diet. Timeframes for return to normal diet varied. $25 \%$ of patients had immediate return to normal diet, $57 \%$ within 3 months, $15 \%$ within 6 months to a year and $2.5 \%$ took over 1 year. A total of 9 patients in this study required a PEG during treatment. One patient surveyed was still using their PEG, 8 years after completing radiotherapy. Two thirds of the patients who required a PEG during their treatment had it removed within 3 months of completing radiotherapy.

\begin{tabular}{|c|c|c|c|c|c|c|c|c|c|}
\hline \multirow[b]{2}{*}{ Tumour site } & \multirow[b]{2}{*}{ Total } & \multicolumn{4}{|c|}{ Significant side effects in the $6 \mathrm{mths}$ post $\mathrm{rt}$} & \multicolumn{4}{|c|}{ Significant side effects 1 year or more post $\mathrm{rt}$} \\
\hline & & Dry mouth & Sore mouth/throat & Dysphagia & Taste changes & Dry mouth & Sore mouth/throat & Dysphagia & Taste changes \\
\hline Larynx & 22 & $41 \%$ & $23 \%$ & $18 \%$ & $14 \%$ & $18 \%$ & $14 \%$ & $9 \%$ & $9 \%$ \\
\hline Oropharynx & 10 & $100 \%$ & $60 \%$ & $60 \%$ & $70 \%$ & $50 \%$ & $30 \%$ & $40 \%$ & $50 \%$ \\
\hline Parotid & 7 & $100 \%$ & $14 \%$ & $29 \%$ & $57 \%$ & $43 \%$ & $14 \%$ & - & $14 \%$ \\
\hline Thyroid & 4 & $50 \%$ & $50 \%$ & $50 \%$ & - & - & - & - & - \\
\hline Neck & 3 & $100 \%$ & $67 \%$ & $100 \%$ & $100 \%$ & $33 \%$ & - & - & $33 \%$ \\
\hline Hypopharynx & 2 & $50 \%$ & $50 \%$ & $50 \%$ & $50 \%$ & - & - & - & - \\
\hline Mandible & 2 & $50 \%$ & $50 \%$ & - & $50 \%$ & $50 \%$ & - & - & - \\
\hline Nasal & 2 & $50 \%$ & - & - & - & - & - & - & - \\
\hline Nasopharynx & 1 & $100 \%$ & $100 \%$ & $100 \%$ & $100 \%$ & $100 \%$ & $100 \%$ & $100 \%$ & - \\
\hline Oral cavity & 1 & $100 \%$ & $100 \%$ & - & - & - & - & - & - \\
\hline
\end{tabular}

From the above table of side effects, dry mouth/xerostomia was reported as the most significant side effect by patients. Over a quarter of patients continued to experience similar levels of xerostomia one year or more after radiotherapy. Patients who had radiation to the mandible, oropharynx and parotid reported to suffer the most from dry mouth. Dysphagia and taste changes/dysgeusia were reported as significant long term side effects for patients who underwent radiation to the oropharynx.

This questionnaire will help to identify the most significant nutrition related problems patients are experiencing after they complete radiation therapy. The findings of this study will be used to facilitate the development of specific guidelines for the nutritional management of this patient group after they complete radiotherapy and aid in the dietetic follow up of this vulnerable patient group.

1. Johnston CA, Keane TJ, Prudo SM (1981) Journal of Parenteral and Enteral Nutrition 6, 399-402.

2. Machtay M et al. (2002) Organ preservation therapy using induction plus concurrent chemoradiation for advanced resectable oropharyngeal carcinoma: a university of Pennsylvania phase II trial. J Clin Oncol 20, 3964-3971.

3. Shilley S et al. (2006) Swallowing function after chemoradiation for advanced stage oropharyngeal cancer. Otolaryngol Head Neck Surg 134, 455-459. 